$\Delta$ Palabras clave/ Local comercial, mercado inmobiliario, precios implíitos.

$\Delta$ Keywords/ business outlet, real estate market, implicit prices.

$\Delta$ Recepción/ 9 octubre 2015

$\Delta$ Aceptación/ 11 enero 2016

\section{Demografía de locales comerciales en la ciudad de Córdoba (España) y determinantes en su valoración.}

Demography of business outlets in the city of Cordoba (Spain) and determinants of their assessment.
Francisco José Rey-Carmona

Doctor en Métodos Cuantitativos en

Economía por la Universidad de Córdoba

(UCO) Córdoba España.

Profesor del Departamento de Estadistica y Organización de Empresas de la UCO, España

td1recaf@uco.es

\section{Julia M. Núñez-Tabales}

Doctora en Métodos Cuantitativos en Economía por la Universidad de Córdoba (UCO), Córdoba, España.

Profesora del Departamento de Estadística y Organización de Empresas de la UCO, España es2nutaj@uco.es

\begin{abstract}
RESUMEN/ Son escasos los estudios empíricos existentes en torno al local comercial, a pesar de su relevancia en la estructura y en la configuración de la ciudad. El funcionamiento de este mercado inmobiliario presenta importantes diferencias con respecto al mercado inmobiliario de uso residencial. El presente artículo analiza el mercado de locales comerciales de la ciudad de Córdoba (España), incluyendo una panorámica general de los establecimientos de la ciudad. Con el objetivo de determinar el precio del local comercial, se ha obtenido un modelo econométrico -haciendo uso de la Metodología de Precios Hedónicos-, lo que ha permitido estimar el peso marginal de cada uno de los atributos que explican el precio total, cuantificando esa influencia de modo que se consiga valorar su importancia relativa sobre el mismo. ABSTRACT/ There are few empirical studies concerning business outlets, in spite of their relevance in a city's structure and setup. This real estate market is quite different from residential real estate markets. This paper discusses business outlet markets in the city of Córdoba (Spain), including an overview of the cities' facilities, in order to determine the price of the business outlets. An econometric model has been developed -using the Hedonic Price Methodology- which has helped estimate the marginal price of each of the features that explain the total price, quantifying such influence so as to value its relative importance on such price.
\end{abstract}

\section{INTRODUCCIÓN}

inmobiliario, el mercado de a vivienda es el principal componente, al punto de que con relativa frecuencia se utilizan ambos términos indistintamente. En consecuencia, la vivienda ha tenido un papel preponderante en la literatura relativa al mercado inmobiliario, mientras que aquel ocupado por el resto de submercados inmobiliarios ha sido marginal. Sin embargo, si se desea tener un mayor conocimiento de la realidad del mercado inmobiliario urbano, se hace necesario establecer un objetivo más ambicioso, ampliando el objeto de estudio a otro tipo de productos inmobiliarios.

En la presente investigación, de entre la amplia tipología de inmuebles existentes, se ha optado por elegir al local comercial como el tipo de inmueble que constituirá el objeto de estudio. Dos son las razones básicas que justifican la elección del local comercial. En primer lugar, la escasez de estudios empíricos existentes en torno a este tipo de inmueble, a pesar de su relevancia en la estructura y en la configuración de la ciudad. En segundo lugar, el local comercial es uno de los tipos de inmueble que más frecuentemente son objeto de valoración, siguiendo a la vivienda.
Por consiguiente, en el presente trabajo se pretende ofrecer una perspectiva general de la demografía de locales comerciales, aplicando a la actividad de las unidades empresariales, como empresas y establecimientos, los conceptos clásicos de los movimientos demográficos referidos tradicionalmente a la población (nacimientos, muertes, migraciones, etc.). Por otro lado, también se efectúa una aproximación a las principales variables que determinan el precio del local comercial. El marco en el que se desarrolla este estudio es la ciudad de Córdoba, España. 


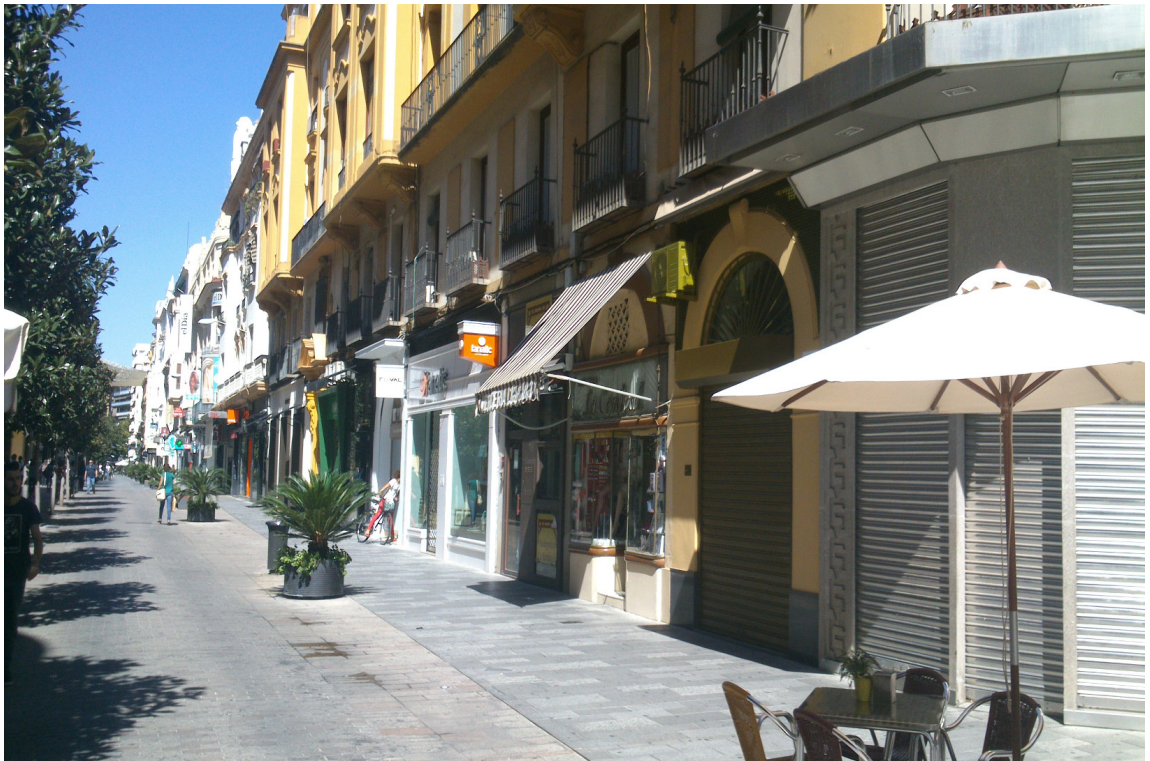

Imagen 1. Céntrica calle comercial José Cruz Conde de la ciudad de Córdoba (fuente: Francisco Rey-Carmona, 2015).

\section{EL FUNCIONAMIENTO DEL MERCADO DE LOCALES COMERCIALES.}

El análisis de los valores de mercado de los locales comerciales debe realizarse en el marco de la situación de los usos de compra de las ciudades donde se sitúan (Caballer, Dos Anjos Ramos y Rodríguez, 2002).

El funcionamiento del mercado inmobiliario de los locales comerciales tiene importantes diferencias con el funcionamiento del mercado inmobiliario de uso residencial (Grajal, 1992). Así, mientras que el uso residencial cubre una necesidad vital del hombre, estando influido de forma directa por el poder adquisitivo de la población y el número de personas que corresponde a cada nivel de renta, esa influencia solo actúa de forma indirecta en el uso terciario. El aspecto de la localización ha sido abordado en la literatura por numerosos estudios, entre los que destacan los realizados por Larraz (2004) en la ciudad de Toledo, y aquellos llevados a cabo por Humarán, Marmolejo y Ruiz (2008) en distintas ciudades catalanas. En imagen 1 se recoge una perspectiva de los locales ubicados en una de las calles con mayor precio de alquiler de la ciudad de Córdoba.
Los inmuebles dedicados al desarrollo de actividades económicas se caracterizan por la gran heterogeneidad que presentan en cuanto a superficie y forma, siendo, en muchas ocasiones, productos que podrían calificarse de residuales, ubicados en las plantas bajas de edificaciones que se han ido construyendo para usos residenciales, tal como se muestra en imagen 2. Por lo tanto, en general, a diferencia de lo que ocurre con los inmuebles residenciales, no son el resultado de programas diseñados de acuerdo a las necesidades de la demanda (Alcat y García, 2006). También es posible encontrar las denominadas 'casas de altos', propias de determinadas áreas geográficas como Chile, consistentes en una vivienda de dos o más niveles. En estos casos, la primera planta alberga funciones comerciales, mientras las plantas superiores son utilizadas con fines habitacionales. En estos inmuebles existe una combinación de funciones -comercio y vivienda- que pueden coexistir de modo independiente, aunque en su origen se encuentra la idea de que es el lugar en donde simultáneamente se vive y se trabaja (Cerda, 2014).

\section{EL MERCADO DE LOCALES COMERCIALES EN LA CIUDAD DE CÓRDOBA (ESPAÑA).}

Desde el inicio de la crisis económica en 2008, el número de locales con actividad no ha dejado de disminuir en España, habiéndose reducido su número en un 6,86\% en el período 2008-2013 según datos del Instituto Nacional de Estadistica. Por su parte, la ciudad de Córdoba no ha estado ajena a dicho fenómeno, pudiendo cuantificarse la caída en el número de locales con actividad en un 7,03\% en el mismo período, porcentaje inferior al de la media de Andalucía (8,4\%). En imagen 3 puede apreciarse un ejemplo de local cerrado en pleno centro de la ciudad. Como puede apreciarse en la tabla 1, la mayor parte de los establecimientos corresponde al sector servicios (imagen 4), representando el $84,52 \%$ del total. Un análisis de la evolución del número de establecimientos por actividades (tabla 1) pone de manifiesto la evolución negativa de casi todas las ramas de actividad, con excepción de 'Información y comunicaciones', 'Banca y seguros' y 'Servicios sanitarios, educativos y restos de servicios' 


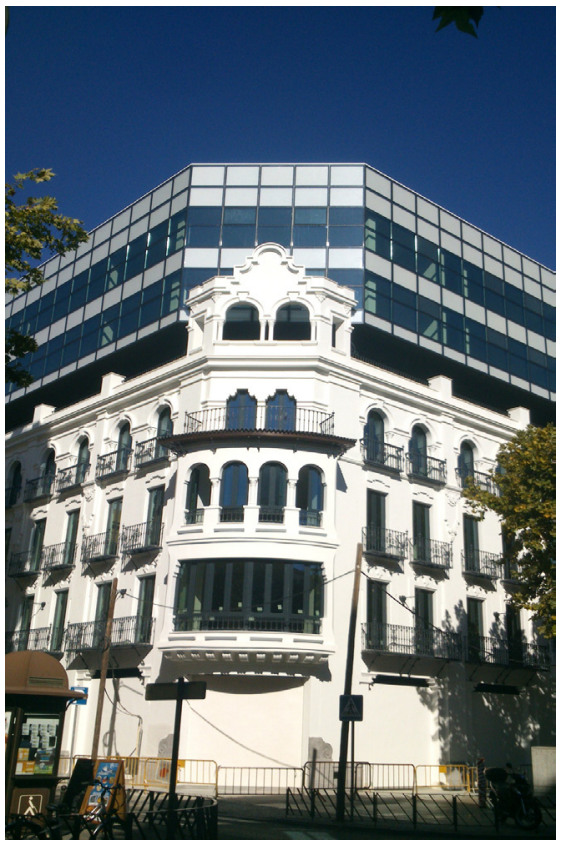

Imagen 2. Edificio en construcción con local comercial en planta baja (fuente: Francisco Rey-Carmona, 2015).

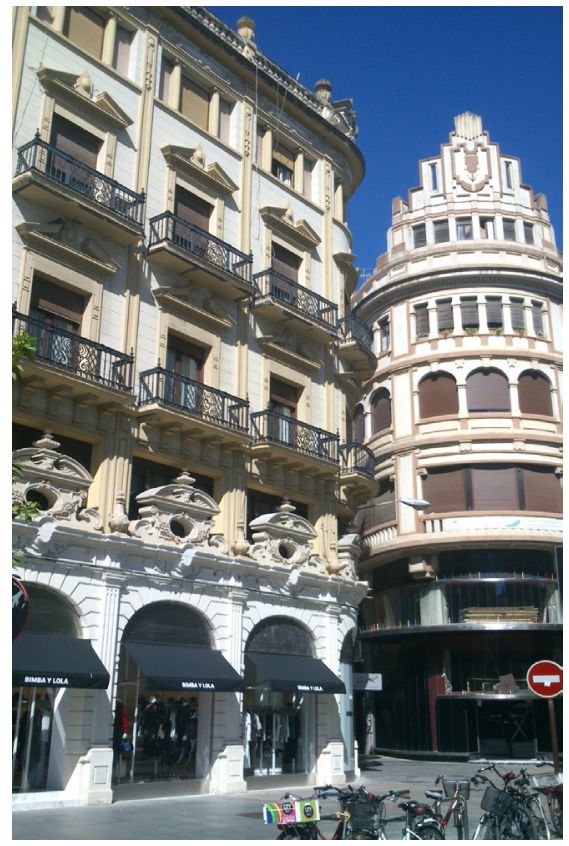

Imagen 3. Locales comerciales situados en la turística Plaza de las Tendillas (fuente: Francisco Rey-Carmona, 2015).

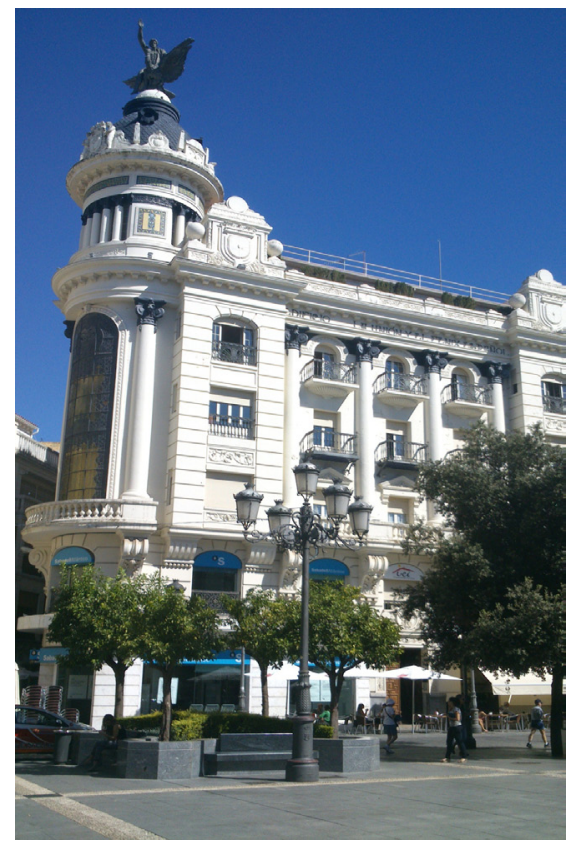

Imagen 4. Edificio con local comercial en planta baja, ocupado por entidad bancaria (fuente: Francisco Rey-Carmona, 2015).
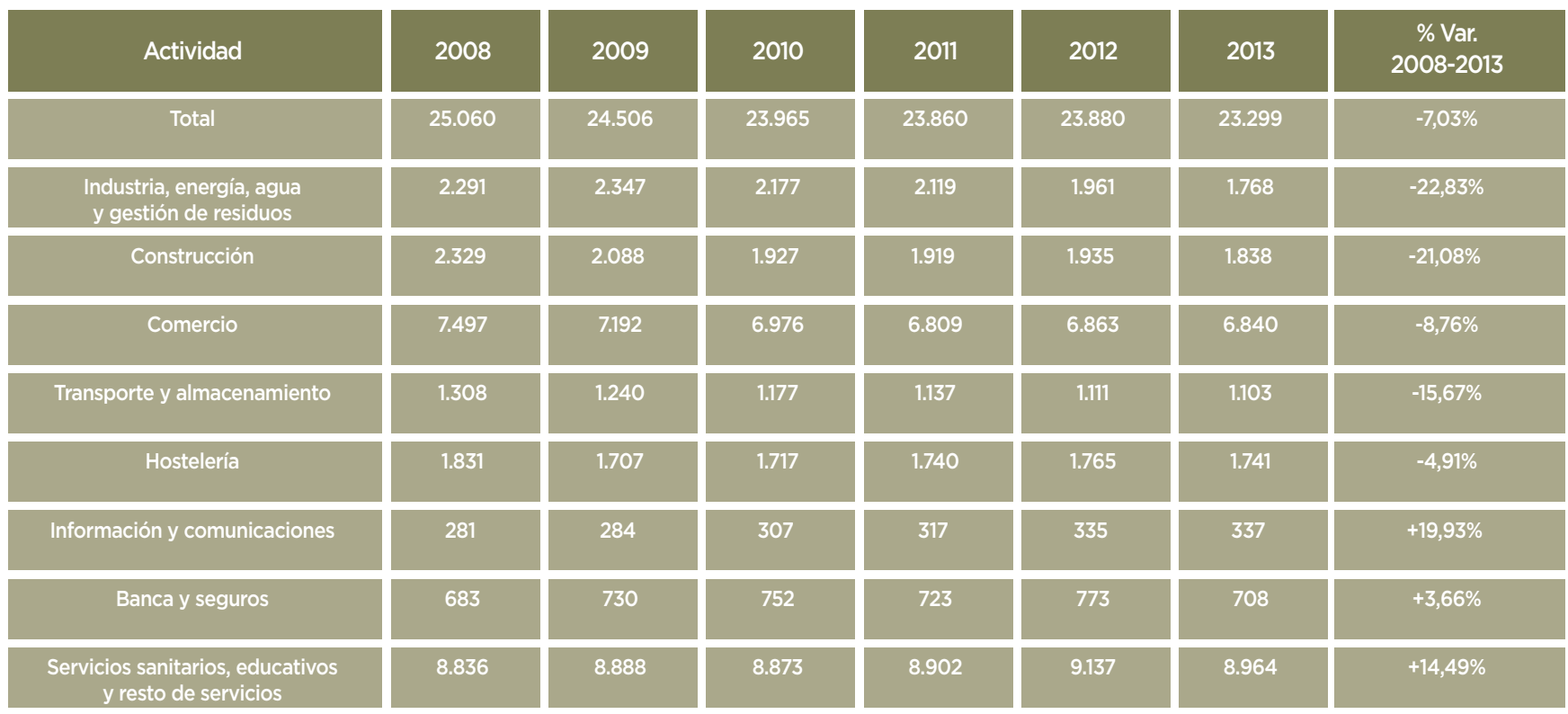

\begin{tabular}{|c|}
\hline 1.308 \\
\hline 1.831 \\
\hline 281
\end{tabular}

\begin{tabular}{|c|}
\hline 1.240 \\
\hline 1.707 \\
\hline
\end{tabular}

\begin{tabular}{|l|}
\hline 1.177 \\
\hline 1.717 \\
\hline 307
\end{tabular}

683

\begin{tabular}{|l|}
\hline 284 \\
\hline 730 \\
\hline 8.888 \\
\hline
\end{tabular}

\begin{tabular}{|l|}
\hline 307 \\
\hline 752 \\
\hline 8.873 \\
\hline
\end{tabular}

y resto de servicio

8.836

8.888

8.873

\begin{tabular}{|c|}
\hline 23.860 \\
\hline 2.119 \\
\hline 1.919 \\
\hline 6.809 \\
\hline
\end{tabular}

\begin{tabular}{|l|}
\hline 1.137 \\
\hline 1.740 \\
\hline
\end{tabular}

\begin{tabular}{|l|}
\hline 1.111 \\
\hline 1.765 \\
\hline
\end{tabular}

\begin{tabular}{|l|}
\hline 1.103 \\
\hline 1.741 \\
\hline
\end{tabular}

$-15,67 \%$

\begin{tabular}{|l|}
\hline 317 \\
\hline 723 \\
\hline
\end{tabular}

8.902

\begin{tabular}{|l|}
\hline 335 \\
\hline 773 \\
\hline
\end{tabular}

\begin{tabular}{|l|}
\hline 337 \\
\hline 708 \\
\hline
\end{tabular}

$-4,91 \%$

\begin{tabular}{|r|}
\hline$+19,93 \%$ \\
\hline$+3,66 \%$ \\
\hline$+14,49 \%$
\end{tabular}

9.137

8.964

$+14,49 \%$

Tabla 1. Evolución de establecimientos por actividades en Córdoba, periodo 2008-2013 (fuente: Elaboración propia a partir del Instituto de Estadística y Cartografía de Andalucia). 


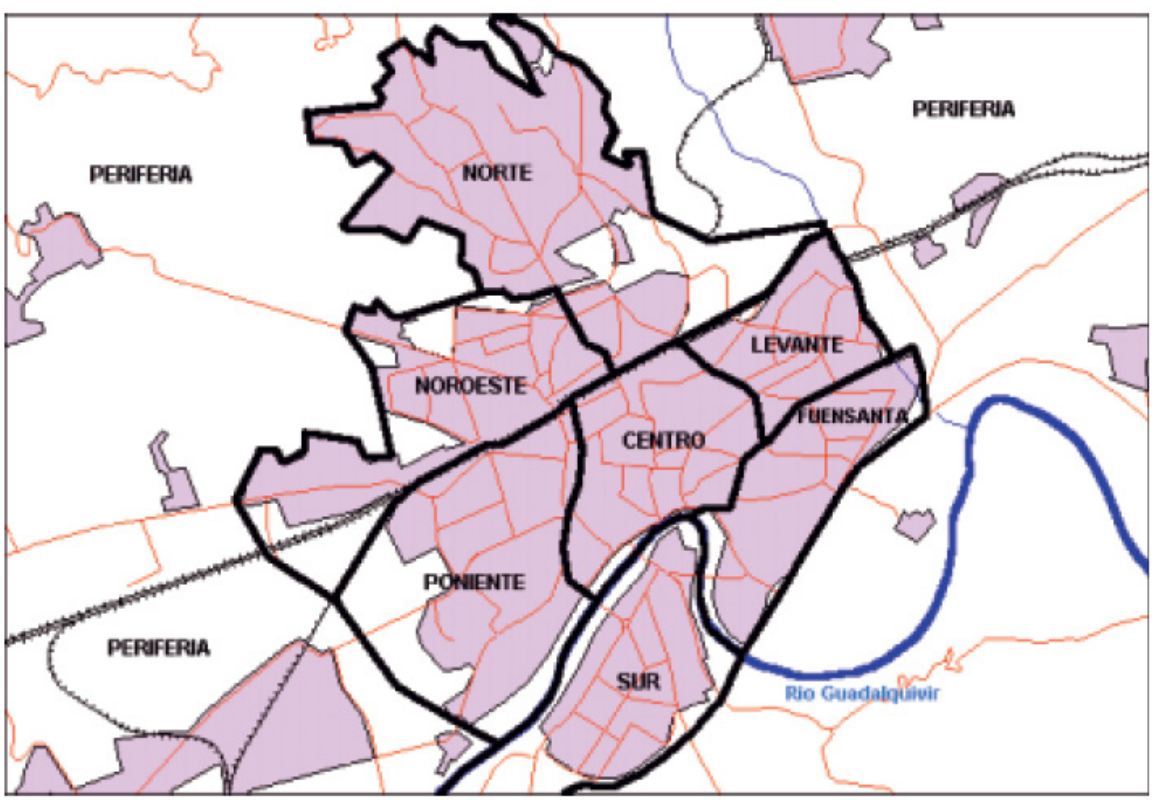

No obstante, la grave crisis económica no ha tenido la misma incidencia en todas las zonas de la ciudad (figura 1). Las reducciones más importantes se observan en la zona sur, en la zona de Levante y especialmente en Fuensanta, mientras que los incrementos en el nivel de actividad se han producido en las zonas noroeste y norte de la ciudad (tabla 2)

En imagen 5 se aprecia la singularidad de los locales comerciales ubicados en la parte antigua de la zona centro de la ciudad.

Figura 1. División por zonas vecinales del municipio de Córdoba (fuente: Anuario Estadístico de la ciudad de Córdoba, 2004)

\begin{tabular}{|c|c|}
\hline $\begin{array}{c}\text { Zona } \\
\text { Vecinal }\end{array}$ & $\begin{array}{c}\text { № Actividades } \\
1-1-2009\end{array}$ \\
\hline CENTRO & 6.660 \\
\hline PONIENTE & 1.997 \\
\hline SUR & 2.479 \\
\hline FUENSANTA & 2.129 \\
\hline NORTE & 3.238 \\
\hline NOROESTE & 4.514 \\
\hline LEVANTE & 5.779 \\
\hline
\end{tabular}

Tabla 2. Evolución de la actividad económica en Córdoba por zonas Vh
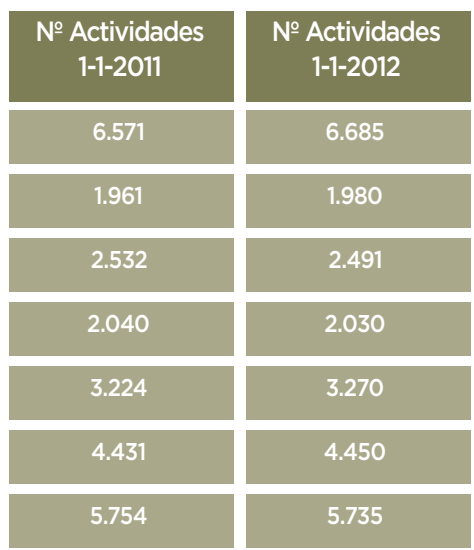

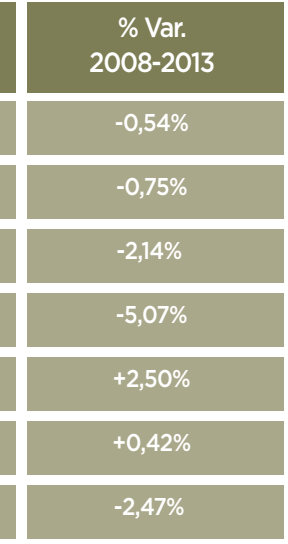

\section{DETERMINANTES DEL PRECIO DEL LOCAL COMERCIAL EN LA CIUDAD DE CÓRDOBA.}

Este apartado tiene como objetivo la obtención de un modelo predictivo del precio mensual de alquiler de un local comercial en la ciudad de Córdoba, ubicado en una zona urbana no periférica. Otros estudios que han utilizado precios de alquiler como variable dependiente del modelo de valoración son los desarrollados por Peña y Ruiz Castillo (1984), Kim (1992) y 올 Stumpf y Torres (1997).
Para ello se seguirá la Metodología de Precios Hedónicos. El Método Hedónico implica que el producto debe tener características identificables por compradores y vendedores, las que pueden variar, y estas variaciones ocasionan cambios en el precio. Estas características son las variables explicativas del modelo matemático (Gallego, 2008). Lo que se pretende, por tanto, es analizar los precios de bienes heterogéneos en base a las características o atributos (internos y externos) que componen las variedades del bien.
Ridker y Henning (1967) fueron los primeros en utilizar dicha metodología en el mercado de la vivienda, en su estudio sobre la incidencia de la polución y las características del vecindario en el precio de la vivienda en San Luis, Estados Unidos. Posteriormente, fueron los trabajos de Griliches (1971) y Rosen (1974) los que difundieron la aplicación de este método. Por su parte, Freeman proporcionó, en 1979, la que muchos autores consideran como la primera justificación teórica para la aplicación de esta metodología al mercado de la vivienda. 


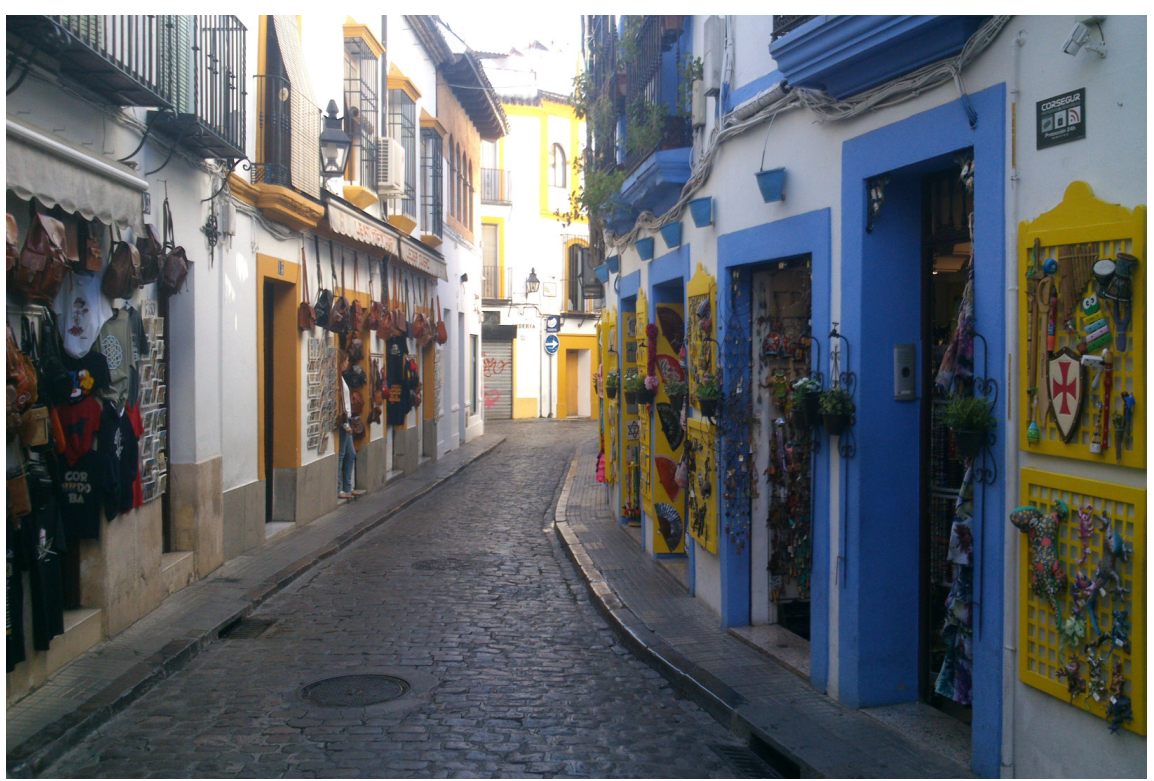

Imagen 5. Típicos locales comerciales situados en el barrio de la Judería (fuente: Francisco Rey-Carmona, 2015).

En el mercado de inmuebles comerciales, es oportuno destacar las aportaciones de Monson (2009), Özyurt (2014) o Deschermeier, Voigtländer y Seipelt (2014). No obstante, en España solo se ha hallado la aportación de Humarán et al. (2008), quienes analizan el precio de los locales comerciales en las principales ciudades catalanas.

Dado que no existen fuentes de información secundarias que pudieran ser consideradas de utilidad para la realización del presente estudio, se ha recurrido a fuentes de información primarias para su elaboración, obteniendo una muestra compuesta por 102 testigos, que abarca la totalidad de las zonas comerciales definidas para la ciudad. En el cuestionario diseñado inicialmente se recogían 38 variables, de las cuales únicamente fueron seleccionadas las 17 que que se encuentran recogidas en la tabla 3 . Con el objetivo último de complementar la información obtenida, se consideró de interés incluir en la base de datos las siguientes variables: zona vecinal, renta de la zona, código postal, número de actividades en cada código postal, número de actividades en cada zona, población en cada zona y densidad de población por actividad en cada zona.
Para trabajar con variables de carácter cualitativo, se construyeron índices que incluyen simultáneamente varios atributos. Estos índices toman valores que oscilan entre 0 y 1 (intervalo cerrado), con el objeto de homogeneizarlos. De esa manera, se pone de manifiesto una situación más favorable a medida que se va aproximando el índice a la unidad. En concreto, los tres índices construidos fueron:

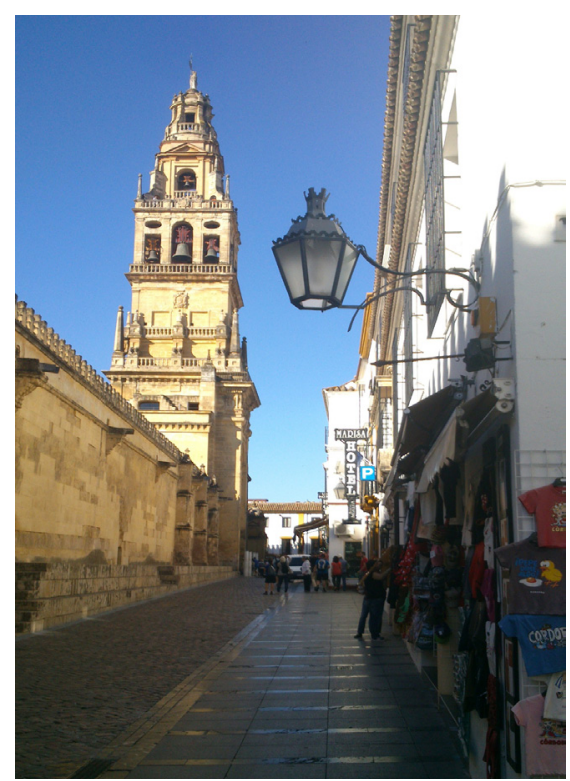

Imagen 6. Local comercial próximo a la Mezquita-Catedral, de excelente ubicación dentro de su barrio y situado en esquina (fuente: Francisco Rey-Carmona, 2015)

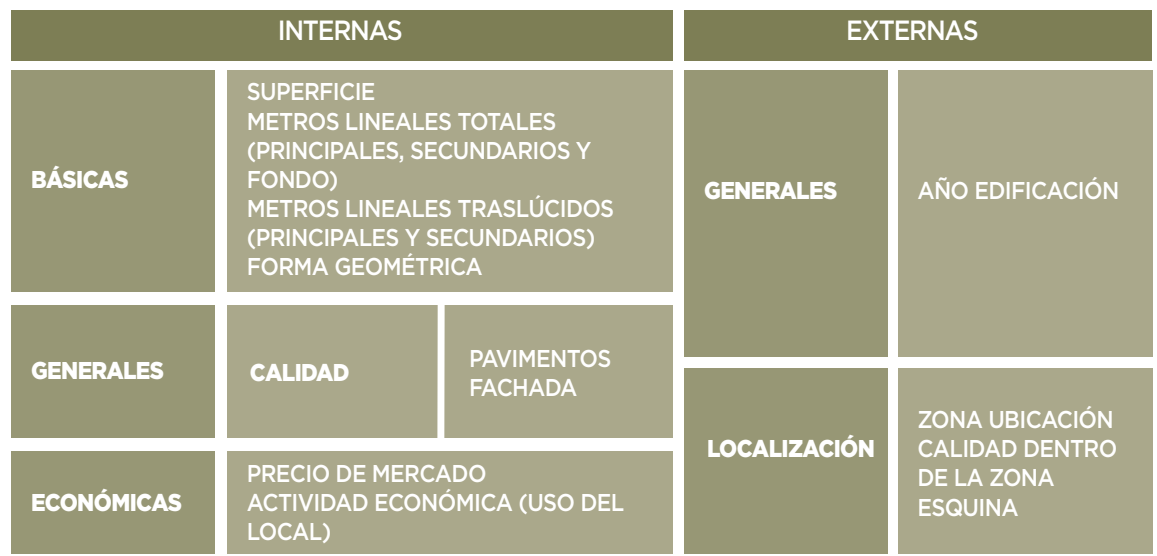

Tabla 3. Clasificación definitiva de variables de los locales de la muestra (fuente: Elaboración propia).

- Índice de Conservación: Para su elaboración se han tenido en cuenta e estado y las calidades tanto de la fachada como del pavimento del mismo.

- Índice de Visualización: Para su construcción se ha incluido la calidad de ubicación del inmueble dentro del barrio y la posición en esquina (imagen 6).

- Índice de Ubicación: Se ha tenido en cuenta el nivel de renta correspondiente a la zona de ubicación del inmueble y la posición geográfica del barrio dentro de la ciudad. 


\begin{tabular}{|c|c|c|c|c|}
\hline VARIABLE & COEFICIENTE & $\begin{array}{c}\text { ERROR } \\
\text { ESTÁNDAR }\end{array}$ & $\begin{array}{l}\text { ESTADÍSTICO } \\
\text { T-STUDENT }\end{array}$ & PROB. \\
\hline c & -1836.681 & 577.1643 & -3.182250 & 0.0020 \\
\hline SUPERFICIE & 6.385094 & 0.768831 & 8.304936 & 0.0000 \\
\hline ANTIGÜEDAD & 20.55353 & 6.823041 & 3.012370 & 0.0034 \\
\hline IUBICACION & 1378.696 & 404.3511 & 3.409650 & 0.0010 \\
\hline CALUBIC^ESQUINA & 137.5182 & 56.23016 & 2.445631 & 0.0165 \\
\hline ICONSERVACION & 884.3945 & 310.6777 & 2.846662 & 0.0055 \\
\hline R-CUADRADO & 0.712318 & \multicolumn{2}{|c|}{ MEDIA DE LA VARIABLE DEPENDIENTE } & 1257.126 \\
\hline R-CUADRADO AJUSTADO & 0.695395 & \multicolumn{2}{|c|}{ CUASI DESV.TÍPICA VAR.DEPENDIENTE } & 1440.676 \\
\hline CUASI DESVIACIÓN TÍPICA RESIDUAL & 795.1242 & \multicolumn{2}{|c|}{ CRITERIO INFORMACIÓN AKAIKE } & 16.25853 \\
\hline SUMA DE CUADRADOS DEL ERROR & 53738913 & \multicolumn{2}{|c|}{ CRITERIO DE SCHWARZ } & 16.42408 \\
\hline LOG. MÁXIMA VEROSIMILITUD & -733.7633 & \multicolumn{2}{|c|}{ ESTADISTIICO F } & 42.09292 \\
\hline & & \multicolumn{2}{|c|}{ PROB (F-STATISTIC) } & 0.000000 \\
\hline
\end{tabular}

Tabla 4. Estimación de la ecuación hedónica (fuente: Elaboración propia).

Prêcio $=-1836.681+6.385094$ Superficie +20.55353 Antigüedad + 1378.696IUbicación +137.5182 CalUbic $^{\wedge}$ Esquina +884.3945 IConservación

Como se ha indicado anteriormente, la variable a explicar es el precio de alquiler de un local comercial. Las características de la ecuación hedónica finalmente obtenida se concretan en la tabla 4. Para su estimación se han utilizado los paquetes EVIEWS $8.0 \mathrm{y}$ SPSS 22.0.

La forma funcional del modelo se reduce a una cuestión empírica. Hay que contemplar la posibilidad de que el modelo recoja interacciones entre las variables, puesto que la oferta de un inmueble no es aditiva. Dicho modelo fue validado tanto de forma global como para cada uno de sus parámetros - de forma individual-, obteniéndose un coeficiente de determinación $\left(R^{2}\right)$ de $71,23 \%$. También se analizó la estabilidad de los parámetros, obteniéndose que el modelo propuesto es estable. De la misma forma, se descartó la presencia de multicolinealidad y se calcularon otros estadísticos para descartar colinealidad.

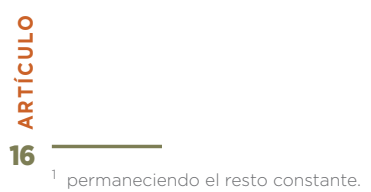

Las variables explicativas de la ecuación hedónica y sus precios implícitos marginales son los siguientes:

- Superficie. Mide las dimensiones del inmueble y está expresada en metros cuadrados construidos. El precio del local se incrementa en 6,385 € cuando la superficie del inmueble aumenta en un metro cuadrado construido, manteniendo constante el resto de variables explicativas. - Antigüedad. Recoge el número de años que tiene el edificio en el que se ubica el inmueble. El precio del inmueble aumenta en 20,55 € por cada año de antigüedad del edificio, manteniendo constante todo lo demás.

- Ubicación. Índice de ubicación del inmueble, que pondera la situación geográfica del inmueble (barrio de la ciudad) junto con el nivel de renta de la zona. Por cada aumento de 0,1 en el indice de ubicación -por la mejora de la situación geográfica del local y/o traslado del mismo hacia una zona catalogada con un nivel de renta mayor- el precio del local se incrementa en 137,8696 €-caeteris paribus'-.

- Calubic^Esquina. Interacción entre la calidad de ubicación del local dentro del barrio concreto en que se ubica y la situación en esquina. La interacción entre la calidad de la ubicación dentro del barrio y la posición en esquina muestra un precio implícito positivo que asciende a 137,5182 €. Es decir, la concurrencia de dichos factores da lugar a incrementos en el precio del inmueble en la citada cifra, manteniendo como en los casos anteriores todas las demás variables constantes.

- IConservación. Índice de conservación, que recoge el estado y calidades de la fachada y el pavimento del inmueble. Cuando el local presenta un estado y calidades tanto de la fachada como de la solería calificado como de "excelente" (índice de conservación $=1$ ), entonces su precio se incrementará en 884,3945 €, a igualdad del resto de elementos. 


\section{DISCUSIÓN Y CONCLUSIONES.}

Como no podía ser de otra manera, la ciudad de Córdoba se ha visto afectada por la grave crisis económica por la que ha atravesado España, experimentando una reducción superior al 7\% en el número de locales con actividad en el período 20082013. Por otro lado, paralelamente se ha puesto de manifiesto un desplazamiento de la actividad económica hacia el norte de la ciudad y en la dirección este a oeste.

La característica que más influye en la determinación del precio de alquiler del local comercial es un factor estructural: la superficie. De hecho, se comprobó que esta variable exógena -por sí sola- era capaz de conseguir un grado de ajuste del $50 \%$, explicando la mitad de la varianza de los valores de mercado de renta de los locales comerciales analizados.

Asimismo, es necesario resaltar la relevancia que para el mercado de locales poseen algunas características de localización. El impacto de las mismas es doble: en primer lugar, se ha observado un comportamiento muy heterogéneo de los precios de alquiler en las diferentes zonas de la ciudad analizadas; en segundo lugar, la calidad de la ubicación dentro de la zona y su situación en esquina también han demostrado tener una enorme incidencia en su precio de alquiler

En concreto, en la ecuación hedónica obtenida se incluyen dos referencias a la localización del inmueble:

1) Macro-localización: Es el índice de ubicación, el cual pondera el barrio en el que se ubica el inmueble dentro de la ciudad y la renta de esa zona.
2) Micro-localización: Viene dada por la interacción entre la calidad de ubicación del inmueble dentro del barrio y si el inmueble se ubica en una esquina.

Cabe destacar la importancia cuantitativa del precio implícito asociado a la macrolocalización del local.

La distinción entre los factores espaciales de macro-localización y micro-localización es ampliamente detallada por López Hernández (2013).

Sin embargo, la presencia de la variable antigüedad fue, en principio, inesperada. Se probaron modelos alternativos que excluían este factor, pero en todos los casos descendía notablemente el grado de ajuste del modelo. En consecuencia, presumiéndose una explicación congruente con esta situación, se encontraron los siguientes argumentos que justifican su presencia:

- Gran parte de los edificios antiguos de la ciudad están ubicados en zonas de cierta tradición o solera comercial en los que, evidentemente, el precio de los locales aumenta.

- Es de esperar que los locales comerciales más antiguos hayan experimentado serias reformas en la fachada, solería, techos, carpintería, por lo que sus calidades generales pueden ser mayores que las de aquellos locales de menor antigüedad.

- En un local comercial no afecta -como en el caso de la vivienda- la antigüedad del edificio. Esto se debe, entre otras razones, a que para acceder a la vivienda hay que atravesar el portal y otras estancias del edificio, mientras que en los locales analizados -todos a pie de calle- no se aprecia el estado y conservación de las zonas comunes del inmueble, ya que se accede al local directamente.

Finalmente, cabe destacar la inclusión de un índice de conservación que recoge la situación de la fachada y la solería del inmueble. Es razonable que el arrendatario esté dispuesto a ofrecer más por el inmueble ante mayores calidades de estos parámetros.

La metodología econométrica y los resultados obtenidos en el presente estudio pueden ser de interés para numerosos colectivos, tanto de naturaleza privada como pública, que deseen aproximarse a la realidad del mercado de locales comerciales. Entre ellos, se pueden citar a los siguientes: ciudadanos inmersos en procesos de valoración inmobiliaria (adquisiciones de viviendas, solicitudes de préstamos hipotecarios, herencias, inversiones en inmuebles, etc.), agentes de la propiedad inmobiliaria, administraciones públicas -puesto que una parte importante de la recaudación tributaria tiene su origen en el gravamen de bienes inmuebles- y empresas de cualquier ámbito económico, de forma especial las pertenecientes al sector financiero, entidades aseguradoras y fondos de inversión inmobiliaria.

Finalmente, cabe señalar que sería conveniente que los propios organismos públicos fomentaran este tipo de investigaciones, dado que permitiría la realización de comparaciones sobre el precio de las transacciones inmobiliarias en diferentes marcos espaciales y temporales, así como de cuáles son los principales determinantes del precio de un inmueble. $\mathbf{\Delta} \boldsymbol{0}$

\section{REFERENCIAS}

Alcat, J. y Garcia M., 2006. Evolución del valor de mercado en locales comerciales 1999-2005, capitales nacionales. Tinsa consultoría.

Ayuntamiento de Córdoba. 2004. Anuario Estadistico de la ciudad de Córdoba.

Caballer, V., Dos Anjo, M. y Rodriguez, J., 2002. El mercado inmobiliario urbano en España. Madrid: Ediciones Pirámide

Cerda, G., 2014. "Casas de altos en el sur de Chile." AUS, 16, 10-14

Deschermeier, P., Voigtländer, M. y Seipelt, B., 2014. Modelling a hedonic index for commercial properties in Berlin. European Real Estate Society (ERES)

Freeman, A., 1979. "Hedonic price, property values and measuring environmental benefits: A survey of the issues." Scandinavian Journal of Economics, 81: 154-173.

Gallego, J., 2008. "Modelos de valoración automatizada." CT: Catastro 62, 7-26.

Grajal, M., 1992. "Valoraciones inmobiliarias de locales comerciales en la ciudad de Valladolid." CT Catastro 13. 31-43.

Griliches, Z., 1971. Introduction: Hedonic Price Indexes Revisited. In Price Indexes and Quality Changes: Studies in New Methods of Measurement. Cambridge: Harvard University Press, 3-15.

Humarán, l:; Marmolejo, C. y Ruiz, M., 2008، "La Formación Espacial de los Valores Comerciales, Un Análisis para las Principales Ciudades Catalanas." (Ponencia), XXXIV Reunión de Estudios Regionales de la Asociación Española de Ciencia Regional, Baeza-Jaén.

Instituto de Estadística y Cartografía de Andalucía (IECA). Recuperado de

http://www.juntadeandalucia.es/institutodeestadisticaycartografia
Instituto Nacional de Estadistica (INE). Recuperado de http://www.ine.es

Kim, S.1992. "Search, hedonic prices and housing demand." The Review of Economics and Statistics, 74 (3), 503-508.

Larraz, B., 2004. Técnicas de cokrigeado para el análisis económico. Estimación de precios de bienes inmuebles en e/ casco histórico de la ciudad de Toledo. (Tesis Doctoral. Universidad de Castilla-La Mancha, España).

López, F., 2013. Sistemas de Información Geográfica y Econometría Espacial en la Tasación de Inmuebles Urbanos. Proyecto Piloto en la Ciudad de Cartagena. Recuperado de http://hdl.handle.net/10317/3138 Monson, M., 2009. "Valuation using hedonic pricing models." Cornell Real Estate Review, 7 (1), $62-73$. Özyurt, S., 2014. "Spatial dependence in commercial property prices: microevidence from the Netherlands" Working Paper Series № 1627. European Central Bank.

Peña y Ruiz-Castillo, J., 1984. "Robust methods of building regression models. An application to the housing sector." Journal of Business and Economic Statistics, 2, 10-20.

Ridker, R. y Henning. A. 1967. "The determinants housing prices and the demand for clean air." Journal Environmental Economy Management, 5: 81-102.

Rosen, S., 1974. "Hedonic prices and implicit markets: product differentiation in pure competition." Journal of Polical Economy, 82: 34-55.

Stumpf, M. y Torres, C. 1997 "Estimación de modelos de precios hedónicos para alquileres residenciales."

Cuadernos de Economía, 101: 71-86. 